\title{
Unruptured Aneurysm of the Sinus of Valsalva With Behçet's Disease
}

\author{
Takanori Kusuyama, MD; Yasuhiro Nakamura, MD; Hiroyuki Yamagishi, MD; \\ Kenei Shimada, MD; Hiroyuki Watanabe, MD; Takashi Muro, MD; \\ Minoru Yoshiyama, MD; Masakazu Teragaki, MD; Kaname Akioka, MD; \\ Kazuhide Takeuchi, MD; Junichi Yoshikawa, MD; Koji Hattori, MD*; \\ Toshihiko Shibata, MD*; Shigefumi Suehiro, MD*
}

\begin{abstract}
A 52-year-old Japanese man who had suffered from Behçet's disease since the age of 45 years was admitted to hospital for evaluation of syncope and heart murmur. Echocardiography and aortography revealed severe aortic regurgitation and cystic masses under the right coronary cusp and the left ventricular outflow tract, but no shunt jet. He was diagnosed with unruptured aneurysm of the sinus of Valsalva, and surgical closure of the orifice of the aneurysm was performed. The diameter of the orifice was $11 \mathrm{~mm}$ and the aneurysm was $15 \mathrm{~mm}$ in depth, and consisted of 2 chambers. Because the aortic regurgitation was reduced after patch closure of the orifice, aortic valve replacement was not performed. Unruptured aneurysm of the sinus of Valsalva is a rare clinical lesion, but patients with active inflammatory disease of the aorta, such as in Behçet's disease, should have periodic echocardiography for early detection of an aneurysm or valvular involvement, even if there are not any symptoms. (Circ J 2002; 66: 107-108)
\end{abstract}

Key Words: Aneurysm; Aortic regurgitation; Behçet's disease; Sinus of Valsalva

A neurysm of the sinus of Valsalva is a rare clinical lesion that is difficult to diagnose because most cases are asymptomatic ${ }^{1-3}$ We describe a rare case of unruptured aneurysm of the sinus of Valsalva associated with Behçet's disease.

\section{Case Report}

A 52-year-old Japanese man who had suffered from Behçet's disease since the age of 45 years was admitted to hospital for evaluation of syncope and heart murmur. The diagnosis of Behçet's disease had been based on oral aphthae, genital ulceration, hyperreactivity of tissues to needle prick and knee joint arthritis, and the patient was considered to have an incomplete form of the disease.

His blood pressure was $102 / 42 \mathrm{mmHg}$ and pulse 72 beats/min. A systolic ejection murmur was maximal on right sternal border in the second intercostal space and an early diastolic murmur was maximal on the left sternal border in the third intercostal space. He had oral aphthae and arthritis of the left knee. A 12-lead electrocardiogram (ECG) revealed sinus rhythm and complete right bundle branch block. Holter ECG, brain X-ray computed tomography and brain magnetic resonance imaging did not detect any other abnormalities.

Transesophageal echocardiography revealed severe aortic

(Received February 22, 2001; revised manuscript received April 20, 2001; accepted May 8, 2001)

Departments of Internal Medicine and Cardiology, and $*$ Cardiovascular Surgery, Osaka City University Graduate School of Medicine, Osaka, Japan

Mailing address: Takanori Kusuyama, MD, Department of Internal Medicine and Cardiology, Osaka City University Graduate School of Medicine, 1-4-3 Asahi-machi, Abeno-ku, Osaka 545-8585, Japan. Email: takanori.kusuyama@nifty.ne.jp regurgitation and 2 cystic masses located under the aortic valve and projecting into the right ventricle (Fig 1). During diastole, the cystic masses expanded and the right coronary

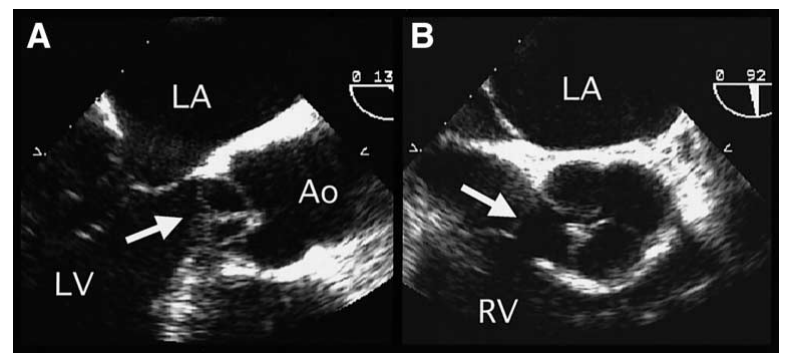

Fig 1. Transesophageal echocardiography. (A) Long-axis view showing 2 cystic masses under the aortic valve (arrow). (B) Shortaxis view showing aneurysm of the sinus of Valsalva projecting into the right ventricle (arrow). Ao, Ascending aorta; LA, left atrium; RV, right ventricle.

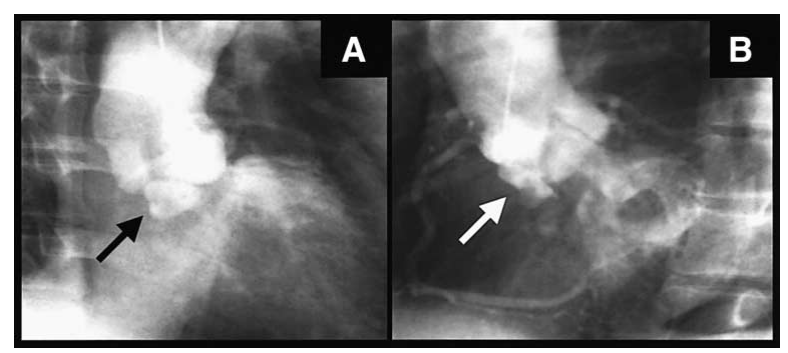

Fig 2. Aortography in the right (A) and left (B) anterior oblique views. There is aortic regurgitation (Sellers grade III), and a cystic mass (arrows) located under the right coronary cusp and in the left ventricular outflow tract. 
cusp prolapsed.

Aortography with contrast medium confirmed the aortic regurgitation (Sellers grade III) and revealed another cystic mass located under the right coronary cusp and in the left ventricular outflow tract (Fig 2). No shunt jet was observed. Coronary arteriography did not detect stenosis. We diagnosed an unruptured aneurysm of the sinus of Valsalva.

Under cardiopulmonary bypass, surgical closure of the orifice of the aneurysm was performed. The orifice of the aneurysm, located in the right coronary sinus through aortotomy, was $11 \mathrm{~mm}$ in diameter and the aneurysm itself was $15 \mathrm{~mm}$ in depth and comprised 2 chambers whose walls consisted of thin membranes. The aneurysm projected into the right ventricle and the left ventricular outflow tract under the right coronary cusp. A horse-pericardial membranous patch was trimmed to $15 \mathrm{~mm}$ in diameter and the orifice was closed with a continuous running 4-0 prolene suture from the aortic side. Because the aortic regurgitation was reduced after closure of the orifice of the aneurysm, aortic valve replacement was not performed. Histological examination of the resected aortic wall revealed productive inflammation, infiltrating inflammatory cells, and moderate atherosclerosis.

\section{Discussion}

The incidence of aneurysm of the sinus of Valsalva ranges from 0.1 to $3.5 \%$ of congenital heart defects, 1 and occurs in $0.14-1.5 \%$ of all patients who have undergone cardiopulmonary bypass?,3 Its occurrence is higher in Asian countries than in the West, ${ }^{1}$ as is the incidence of Behçet's disease.

Behçet's disease is a systemic inflammatory disease, with cardiac manifestations, such as pericarditis, aortic or mitral regurgitation, endomyocardial fibrosis, arterial or venous occlusion, varices and aneurysm of the sinus of Valsalva, occurring in about $5 \%$ of patients. Although the incidence of cardiac manifestations is low, they can have severe clinical consequences. In the present case, there was chronic inflammation of the aorta, which is also seen in other inflammatory conditions (eg, syphilitic aortitis, tuberculous aortitis, bacterial endocarditis, and some collagen diseases) and which makes the aortic wall fragile6 Therefore, the present case of aneurysm was considered to be caused by the Behçet's disease6

Abe et al reported that $94 \%$ of aneurysms of the sinus of Valsalva originate from the right coronary sinus and $6 \%$ originate from the non-coronary sinus? Almost all such aneurysms project into the right atrium or right ventricle, but may rupture into the pulmonary artery, left ventricle, left atrium or the free pericardial cavity2,5 In the present case, the aneurysm projected into the right and left ventricles, and was at risk of rupture into these chambers.

Aneurysms of the sinus of Valsalva have both congenital and acquired etiologies. Congenital causes include insufficient adhesion of the endocardial cushion, and connective tissue diseases such as Marfan's syndrome. Acquired etiologies include chronic inflammatory diseases, autoimmune disease and other conditions.,5

Only 7 cases of aneurysm of the sinus of Valsalva accompanying Behçet's disease have been reported, and only 2 were unruptured., 7 Some asymptomatic cases of ruptured aneurysm of the sinus of Valsalva have been reported 8,9 It is easy to diagnose a ruptured aneurysm because in most cases it causes acute heart failure, but it is difficult to diagnose unruptured aneurysm of the sinus of Valsalva because almost all cases are asymptomatic. The symptoms and signs of aneurysms of the sinus of Valsalva are many, including palpitations, general fatigue, exertional dyspnea, syncope, congestive heart failure, pleural effusion, infectious endocarditis, and conduction disturbance $2,3,6,10-12$ The chief findings in the 2 reported cases of unruptured aneurysm were auscultational changes and low-grade fever, 4 The chief complaint in the present case was an episode of syncope. Abe et al reported that 12 (39\%) of 31 patients with a ruptured aneurysm of the sinus of Valsalva had episodes of syncope? but the reason for syncope in cases of ruptured or unruptured aneurysm of the sinus of Valsalva has not been clearly established. These aneurysms of the sinus of Valsalva are a risk for the development of infectious endocarditis, acute heart failure and thrombosis $1^{13}$ and may worsen valvular disease.

Aneurysms of the sinus of Valsalva caused by active inflammatory disease, such as Behçet's disease, tend to enlarge progressively and have a high risk of rupture. Accordingly, surgical repair should be performed as soon as possible and patients with Behçet's disease should have periodic echocardiography examinations for early detection of either aneurysm or valvular involvement, even if there are not any symptoms.

\section{References}

1. Islam MN, Alimuzzaman M, Khan MNA, Bashar MA, Zafar A. Ruptured aneurysm of the sinus of Valsalva. Bangladesh Med Res Council Bull 1996; 22: 19-26.

2. Abe T, Komatsu S. Surgical repair and long-term results in ruptured sinus of Valsalva aneurysm. Ann Thorac Surg 1988; 46: 520-525.

3. Choudhary SK, Bhan A, Reddy SCB, Sharma R, Murari V, Arian B, et al. Aneurysm of sinus of Valsalva dissecting into interventricular septum. Ann Thorac Surg 1998; 65: 735-740.

4. Okada K, Eishi K, Kitoh Y, Kosakai Y, Isobe F, Nakano K, et al. Huge aneurysm of the sinus of Valsalva following infective endocarditis in Behçet's disease. J Heart Valve Dis 1997; 6: 179-180.

5. Koh KK, Lee KH, Kim SS, Lee SC, Jin SH, Cho SW. Ruptured aneurysm of the sinus of Valsalva in a patient with Behçet's disease. Int J Cardiol 1994; 47: 177-179.

6. Nojiri C, Endo M, Koyanagi H. Conduction disturbance in Behçet's disease: Association with ruptured aneurysm of the sinus of Valsalva into the left ventricular cavity. Chest 1984 ; 86: 636-638.

7. Wakabayashi Y, Tawarahara K, Kurata C. Aneurysms of all sinuses of Valsalva and aortic valve prolapse secondary to Behçet's disease. Eur Heart J 1996; 17: 1766.

8. Chen JJ, Lien WP, Chang FZ, Lee YS, Hung CR, Chu SS, et al. Ruptured congenital aneurysm of the right sinus of Valsalva into the right ventricle: With special reference to pathoanatomic and hemodynamic characteristics in symptomless cases. Jpn Circ J 1980; 44: $87-94$.

9. Suzuki T, Hirose M, Murase K, Baba E, Senda H. Proceedings: Ruptured aneurysm of the Valsalva's sinus. Jpn Circ J 1975; 39: 875.

10. Ahmad RAS, Sturman S, Watoson RDS. Unruptured aneurysm of the sinus of Valsalva presenting with isolated heart block: Echocardiographic diagnosis and successful surgical repair. Br Heart J 1989; 61: $375-377$.

11. Dazai Y, Katoh I, Hara Y, Yoshida R, Joh T, Tomino T. A rare case of infective aneurysm involving all 3 sinuses of Valsalva complicated by left single coronary artery. Jpn Circ J 1991; 55: 159-164.

12. Ishise S, Okuda K, Kumazawa T, Masutani K, Miyoshi K. Proceedings: Rupture of Valsalva's sinus: A case study. Jpn Circ J 1975; 39: 739.

13. Pepper C, Munsch C, Sivananthan UM, Pye M. Unruptured aneurysm of the left sinus of Valsalva extending into the left ventricular outflow tract: Presentation and imaging. Heart 1998; 80: 190-193. 